\title{
Correction to: Comparative Effectiveness and Tolerability of the Pharmacology of Monoclonal Antibodies Targeting the Calcitonin Gene-Related Peptide and Its Receptor for the Prevention of Chronic Migraine: a Network Meta-analysis of Randomized Controlled Trials
}

\author{
Chun-Pai Yang ${ }^{1,2}$ - Bing-Yan Zeng ${ }^{3,14} \cdot$ Ching-Mao Chang $^{4,5,6} \cdot$ Po-Hsuan Shih ${ }^{6,7}$. Cheng-Chia Yang ${ }^{8}$. \\ Ping-Tao Tseng ${ }^{9,10,11} \cdot$ Shuu-Jiun Wang ${ }^{5,12,13}$
}

Published online: 6 October 2021

(c) The American Society for Experimental NeuroTherapeutics, Inc. 2021

\section{Correction to: Neurotherapeutics (2021)}

https://doi.org/10.1007/s13311-021-01128-0
Publisher's Note Springer Nature remains neutral with regard to jurisdictional claims in published maps and institutional affiliations.

The original version of this article was revised to add a second affiliation "Division of Endocrinology \& Metabolism, Department of Internal Medicine, E-DA Dachang Hospital, Kaohsiung, Taiwan" to author Bing-Yan Zeng.

The Original article has been corrected.

The original article can be found online at https://doi.org/10.1007/ s13311-021-01128-0.

Ping-Tao Tseng ducktseng@gmail.com

$\square$ Shuu-Jiun Wang sjwang@vghtpe.gov.tw

1 Department of Neurology, Kuang Tien General Hospital, Taichung, Taiwan

2 Department of Nutrition, Hungkuang University, Taichung, Taiwan

3 Division of Endocrinology and Metabolism, Department of Internal Medicine, E-Da Hospital, Kaohsiung, Taiwan

4 Center for Traditional Medicine, Taipei Veterans General Hospital, Taipei, Taiwan

5 Faculty of Medicine, College of Medicine, National Yang Ming Chiao Tung University, Taipei, Taiwan

6 Institute of Traditional Medicine, National Yang Ming Chiao Tung University, Taipei, Taiwan

7 Department of Chinese Medicine, Cheng Hsin General Hospital, Taipei, Taiwan
8 Department of Healthcare Administration, Asia University, Taichung, Taiwan

9 Prospect Clinic for Otorhinolaryngology \& Neurology, Kaohsiung City, Taiwan

10 Department of Psychology, College of Medical and Health Science, Asia University, Taichung, Taiwan

11 Institute of Biomedical Sciences, National Sun Yat-Sen University, Kaohsiung, Taiwan

12 Department of Neurology, Neurological Institute, Taipei Veterans General Hospital, Taipei, Taiwan

13 Brain Research Center, National Yang Ming Chiao Tung University, Taipei, Taiwan

14 Division of Endocrinology \& Metabolism, Department of Internal Medicine, E-DA Dachang Hospital, Kaohsiung, Taiwan 